\section{Clinital aldetes:}

\section{MEDICAL, SURGICAL, OBSTETRICAL, AND THERAPEUTICAL.}

\section{RECOVERI AFTER COMPOLND FRACTURE OF SKULL WITH EXTRAVASATION OF BRAIN.}

BY T. E. Coulson, M.D., F.R.C.S. EdIN.,

TEMP. LIEU'l'WANT, R.A.M.C.; SURGHON T'O THE MERTHYR GUEST HOSPITAL, TEMPLECOMIBE.

THE following case of compound fracture of the skull with extravasation of brain matter, in which immediate operation resulted in complete recovery, may be of some interest :-

On June 13th, 1918, a boy, aged 9, was riding in a wagon in a hayfield with two girls, when the horse "bolted." One of the girls jumped out over the side of the ragon, the wheel went over her leg, and she sustained a compound fracture-dislocation of the ankle-joint. The otber girl became frightened, seized the child and threw him out of the wagon over the side, both wheels going over his head. She herself then climbed out over the back and escaped unburt.

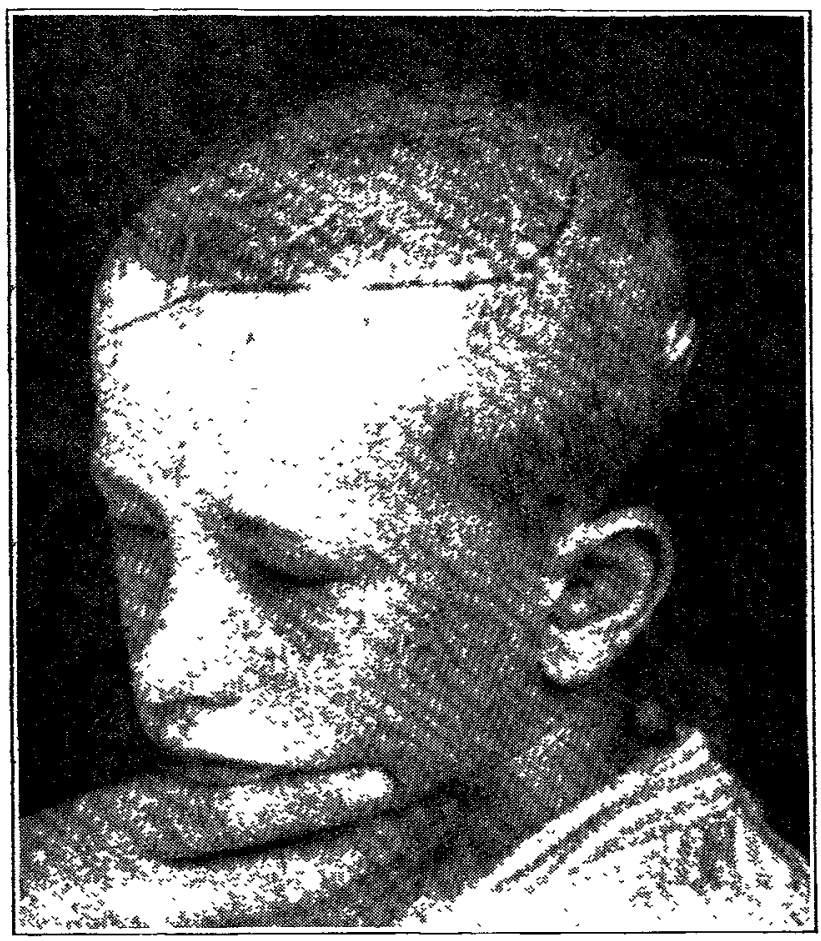

Photograph of patient on June 26th, 13 days after operation.

This happened at about 4 P.M., and the boy was admitted to the Mert hyr Guest Hospital at 4.30. When I saw him at 4.45 he was semi-

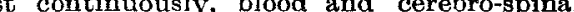
fluid coming freely from both ears. There were two wounds, each about the size of a threepenny-piece, on right side of head, one about 2 inches above right ear and the other about halfway between right ear and right eye; from the former of these brain matter was oozing in considerable quantity. There was a linear depressed fracture on both sides of head running from before backwards. Temperature subnormal; pulse 90 per min.; pupils dilated and equal; no paralysis of face or limbs.

$I$ operated at 5 P.M. After shaving the head and painting it with chloroformum iodi an incision was made from a point 1 in bebind right ear vertically npwards for 3 in, then directly forwards for $5 \frac{1}{1}$ in. to a point 1 in above centre of right eyebrow. This curved incision ran 1 in. above line of depressed fracture on this side. At posterior end this fracture was star-shaped, one "limb" of star pointing downward in front of right ear (from this brain matter was oozing), one pointing behind right ear, one upwards and backwards, and the main fracture forwards to thear, one upwards and back wards, and the main fracture forwards to the point $1 \frac{1}{2}$ in. above centre of right eyebrow. The centre beneath the parietal bone, between it and the dura. It was withdrawn and discarded.

Attention was now turned to the main depressed fracture on this side. The lower fragment was depressed beneath the upper, so that the latter overhung the former by about inch. I therefore cut away with bone forceps a wedge-shaped piece of upper fragment about its centre and, introducing an elevator, lifted up lower fragment on a level with upper. There followed a good deal of hæmorrbage, which, however, ceased in a short time. The fracture on left side extended from the point $1 \frac{1}{2}$ in. above centre of right eyebrow, where fracture on right side ended, backwards to the left to a point $3 \frac{1}{2}$ in. above left ear, and then of this fracture. The illustration show's line of incision on this side.
On the left side upper fragment was depressed beneath lower fragment to extent of about $\frac{1}{2}$ in. I applied a $\frac{3}{4}$ in. trephine to edge of lower fragment at angle above lett ear, and introducing an elevator levered upper fragment into position level with lower fragment. This fracture ended posteriorly in a star-shaped manner, but the "star" was not so marked as on right side, and no brain matter was being extravasated on this side.

The various manipulations described had by now taken some time, and the boy having lost a good deal of blood he began to show signs of shock. The pulse became very weak and fast; it had risen to 130 per minute. Resptrations became "catchy," and would occasionally cease for a considerable time. I had intended to investigate fracture running
in front of right ear from which brain matter was coming away, but it became erident that the boy would not stand any further procedure. so I contented myself with swabbing the brain matter away with an antiseptic and stitching up the whole wound with interrupted fishing gut sutures, without drainage.

For the first day or two patient was in an extremely critical condition, semiconscious, verv violent and irritable when roused; pulse 120 ; temp. subnormal. Calomel gr. ili. was given on the evening after the operation and after this had acted on the following evening he became much quieter and more rational. On June 16 th he was able to rationall control over bladder and rectum. The stitches were removed June 22 nd and all bandages taken off on the 26th. On June 23rd. 24th June 22nd and all bandages taken off on the 26th. On June 23rd, 24th and $25 t h$ there whs very slight watery blood-stained dischar

The boy is now up and about and appears perfectly normal in all respects with the exception of slight deafness in left ear.

\section{RADICAL CURE OF A VERY LARGE INGUINAL HERNIA.}

By C. P. G. WAKELEY, M.R.C.S. ENG., L.R.C.P. LOND. TEMPORARY SURGEON, R.N., H.M. HOSPITAL SHIP "GARTH OASTLE."

Dr. J. A. Browne's case of large inguinal hernia, ${ }^{1}$ which was reported in a recent number of THE LANCET, aroused my interest because $I$ had a very similar case under my care several years ago, when I was house surgeon to Mr. Albert Carless at King's College Hospital. The enormous size of the hernia can be seen from the photograph; it was a left oblique inguinal hernia and descended to the patient's knees, causing great inconvenience in walking. The patient, aged 50 , was a very tall man. He stated that he had noticed the rupture for over 10 years. The hernia could be reduced with diffioulty, but could not possibly be controlled by any form of truss or bandage. The penis was completely hidden,

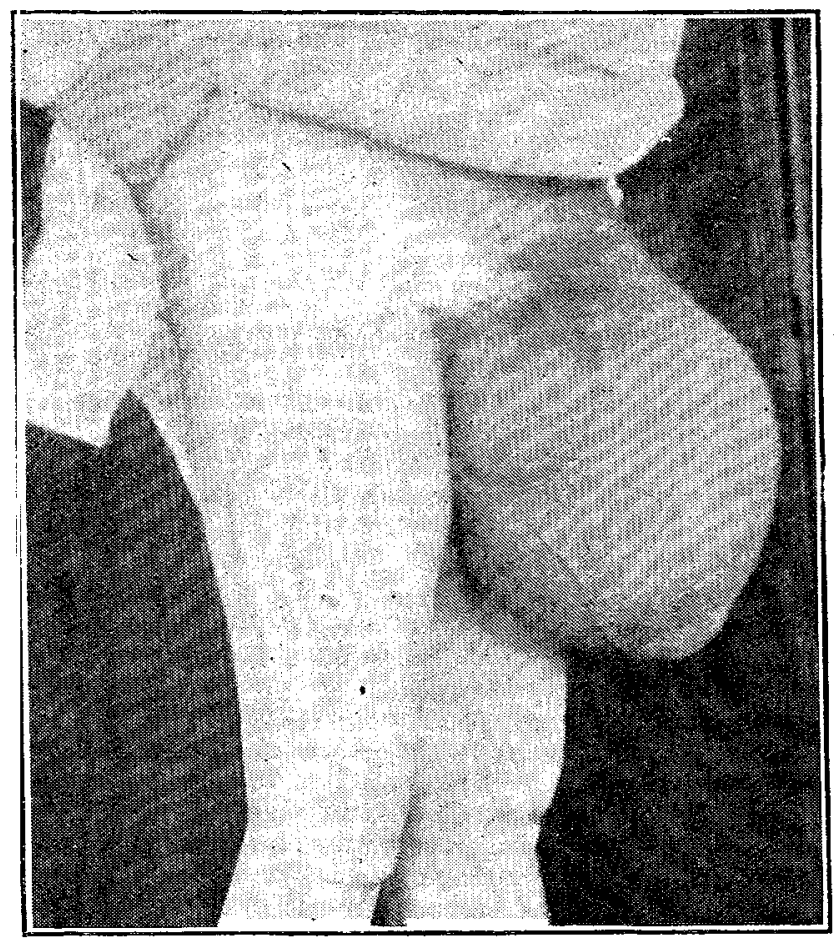

and could only be located at the bottom of a small depression at the upper part of the swelling. Micturition was difficult at times. The opening of the canal admitted four fingers easily. The patient was kept on his back with the foot of the bed raised for two weeks previous to the operation. A radical cure was performed; the left testicle, which 